\title{
CONTRIBUTION A L'ÉTUDE DES EMPLOIS DES ACIERS INOXYDABLES DANS LES LAITERIES ET LES FROMAGERIES
}

\author{
par
}

\author{
J. LEMOINE \\ Ingénieur E. C. P.
}

Les matières premières nécessaires à la construction des machines et appareils utilisés pour le traitement du lait et de ses dérivés doivent répondre à certaines conditions imposées par les problèmes variés et toujours délicats des différents traitements de ces produits.

Le lait, d'une composition chimique complexe, tend à se transformer et il faudra des précautions particulières pour assurer sa conservation. Dans d'autres cas, au contraire, on devra diriger sa transformation pour la fabrication des beurres et des fromages. Il sera done soumis, suivant les cas, à des traitements mécaniques, à des traitements thermiques, à l'action des ferments, etc.

Dans ces conditions, il faudra prévoir des récipients, des tables, des moules, des essoreuses, des tuyauteries, des échangeurs de température, etc.

On fera appel au bois, au grès, au verre, aux divers métaux naturels ou émaillés ou étamés, ou encore aux alliages, bronze, laiton, aciers inoxydables.

Ces matériaux seront en contact plus ou moins prolongé avec les produits en transformation, avec les acides qu'ils contiennent (acide lactique, acide butyrique,...). Ils devront résister à leur action chimique et, d'autre part, aux produits employés pour le nettoyage des appareils.

Enfin, le prix d'achat et la durée d'amortissement devront être acceptables.

Il n'existe aucun matériau qui satisfasse à la fois à toutes ces conditions, attendu que la laiterie, la fromagerie et la beurrerie nous mettent en présence de nombreux problèmes et que chacun d'eux exige des solutions différentes.

Sans faire le procès des autres matières qui sont utilisées pour le matériel de-laiterie, nous nous proposons ici d'examiner seulement le cas des aciers inoxydables. Nous pensons que ces nouveaux venus de la métallurgie ne sont pas encore suffisamment connus du public, bien que leurs propriétés mécaniques et chimiques les désignent tout particulièrement pour la construction d'un grand nombre d'appareils utilisés en laiterie et en fromagerie. Nous allons essayer de montrer l'intérêt incontestable que présente l'emploi de ces aciers, et citer, à titre d'exemple, les applications déjà réalisées. 


\section{Composition des aciers inoxydables}

Parmi les compositions d'aciers inọxydables, on a d'abord songé à utiliser les aciers inoxydables au chrome. Ceux-ci servent encore pour certaines pièces mais présentent le gros inconvénient de n'avoir une bonne résistance à la corrosion qu'après polissage ou rectification très fine de leurs surfaces. Ils se soudent difficilement et ne conviennent pas à l'exécution des pièces chaudronnées.

Les aciers inoxydables à $18 \%$ de chrome et à $8 \%$ de nickel connus sous le nom de "aciers $18 / 8$ " se sont montrés plus intéressants. Ils sont inoxydables même si leur surface est simplement décapée sans être polie. Ils conviennent très bien à l'exécution des appareils et en particulier des pièçes chaudronnées qui constituent une partie importante du matériel de laiterie.

A la suite des recherches des métallurgistes, ces aciers ont subi des modifications de leurs compositions, ayant pour but d'éviter que leur soudure ou leur travail à chaud diminuent leur résistance à la corrosion; de nouveaux "aciers $18 / 8$ » ont done été mis au point et ce sont ces aciers qui sont aujourd'hui adoptés pour le matériel de laiterie, parce qu'ils répondent le mieux aux conditions que nous avons indiquées plus haut.

\section{Résistance à la corrosion}

Ces aciers résistent d'une façon parfaite à de nombreux acides, à ceux du lait et de ses dérivés; enfin, ils ne sont pas attaqués par les différents produits employés dans les laiteries et les fromageries, pour la fabrication (sel marin, par exemple) ou pour le nettoyage ou la désinfection du matériel.

Ils sont autorisés par le Service de la Répression des Fraudes du Ministère français de l'Agriculture, suivant les dispositions du décret du 28 juin 1912, qui réglemente l'emploi des métaux au contact des boissons et des denrées alimentaires.

Le nettoyage des ustensiles en acier 18/8 est particulièrement aisé. Tous les produits courants de nettoyage permettent d'obtenir immédiatement des surfaces absolument propres et exemptes d'aspérités, ce que l'on ne peut éviter avec les ustensiles courants en fer étamé, de sorte qu'au point de vue de l'hygiène, le matériel en acier inoxydable présente un avantage incontestable sur le matériel courant en tôle étamée. Toutefois, il convient d'éviter d'employer, pour ce nettoyage, de l'eau de Javel en solution renfermant plus de 15 milligrammes de chlore libre par litre. 


\section{Caractéristiques mécaniques}

Les caractéristiques mécaniques de ces aciers sont les suivantes : Limite élastique : $\mathrm{E}>25$ kilogrammes par millimètre carré.

Charge de rupture : $\mathrm{R}>60$ kilogrammes par millimètre carré.
Allongement
: $A \%>40$ kilogrammes par millimètre carré.

Ces chiffres sont ceux que l'on obtient sur des éprouvettes de traction de $13 \mathrm{~mm} .8$ de diamètre, $\mathrm{A} \%$ étant mesuré entre deux repères distants de 100 millimètres.

\section{Caractéristiques physiques}

Chaleur spécifique : 0,12 unités C. G. S. entre $0^{\circ}$ et $100^{\circ}$.

Coefficient de dilatation : $16,100^{6}$ entre 0 et $100^{\circ}$.

Conductibilité calorifique : 0,04 unités C. G. S. à $100^{\circ} \mathrm{C}$.

Résistance chimique 70: microhms $\mathrm{Cm} 2 / \mathrm{cm}$.

\section{Produits fabriqués}

Les aciéries livrent ces aciers sous les formes suivantes :

Pièces moulées, pièces forgées, barres et tôles laminées à chaud ou à froid, feuillards laminés à froid, étirés, fils et tubes étirés sans soudure, soit sous toutes les formes nécessaires pour la construction mécanique et la fabrication des appareils chaudronnés.

\section{Fabrication du matériel en acier inoxydable}

Nous ne pouvons pas donner ici d'indications détaillées qui sortiraient du cadre de cette étude; nous indiquons seụlement que ces aciers peuvent s'usiner, s'emboutir, être soudés à l'arc, à l'autogène et par soudure électrique par points ou à la molette. Ils conviennent pour les travaux de chaudronnerie courante. Toutefois, ces différentes opérations de transformation, sans présenter de difficulté, exigent certaines précautions.

Les pièces chaudronnées sont le plus souvent livrées décapées ou plus rarement polies; elles sont dans tous les cas plus légères que les pièces en fer étamé de même forme, pour une même résistance.

Le polissage brillant qu'il est possible d'obtenir a l'avantage de se conserver indéfiniment, sans se ternir, ce qui permet d'avoir des appareils d'une très grande propreté. C'est pourquoi les pièces polies en acier inoxydable sont également d'un emploi assez répandu, bien que le polissage ne soit pas indispensable par lui-même pour la bonne résistance à l'oxydation.

\section{Applications réalisées}

On peut signaler les appareils et ustensiles en acier inoxydable suivants : 
Laiterie : Seaux, bassines, cuves, pots à lait, canalisations, appareils de chauffage et de réfrigération du lait, pasteurisateurs, machines pour le lavage et pour le remplissage des bouteilles à lait, eiternes isothermes de transport, distributeurs de lait pour la vente au détail, appareils pour le traitement du lait dans les condenseries.

Beurrerie : Bacs de maturation, bols et assiettes d'épurateurs centrifuges et d'écrémeuses, tables pour la préparation du beurre, machines à mouler et à empaqueter le beurre(1), machines à remplir les pots à crème.

Fromagerie: Plaques et moules à fromage, tables d'égouttage, cuves de cuisson de fromages (Gruyère, Emmental), machines à mouler et à empaqueter les fromages fondus.

Le procédé de conservation du lait par la méthode Hofius utilise des récipients de 50 litres en acier inoxydable; ces récipients doivent résister à une pression de 10 kilogrammes par centimètre carré.

Les aciers inoxydables doivent également trouver un débouché dans la fabrication des boîtes à lait concentré ou en poudre (il a, en effet, été démontré que l'étain produit une réaction chimique nuisible à la conservation de ces produits).

Comme application probable pour l'avenir, on peut citer les foudres pour le transport direct du lait des centres régionaux dans les établissements de vente au détail des grandes villes.

\section{Amortissement}

Les propriétés de ces aciers inoxydables, que nous venons de rappeler, montrent bien les possibilités qu'ils offrent pour la fabrication du matériel de laiterie, et on peut dire que si jusqu'à ces derniers 'temps l'emploi de ces aciers dans ces applications n'a pas été plus développé, c'est en raison des prix qui ont été pratiqués, et qui étaient justifiés, puisqu'il s'agissait d'aciers nouveaux. Les prix de ces aciers avaient considérablement diminué depuis ees derniers temps, et deviendront sans aucun doute très accessibles dès que la situation sera redevenue normale.

Si on tient compte de ce que les pièces en aciers inoxydables ne périssent que par usure mécanique, sont d'un nettoyage facile, d'un entretien nul et permettent une amélioration du rendement et de la qualité des produits, on démontre qu'elles constituent une réelle f́conomie et s'amortissent dans un temps normal.

(1) Ces machines fonctionnent dans une atmosphère saturée de vapeur d'eau à une température de 18 à $20^{\circ}$; la plus petite trace de rouille déréglerait les cames et immobiliserait ces machines, d'où la nécessité d'employer un acier inoxydable. 